\title{
Factores de riesgo relacionados con estilos de vida asociados a la progresión de cáncer de mama en una población atendida en un establecimiento de salud de nivel 3 de Lima Metropolitana
}

\section{Lifestyle-related risk factors associated with the progression of breast cancer in a population attended in a level 3-health care establishment in Lima}

\author{
${ }^{1}$ Hattie Alderete, ${ }^{1}$ Brayan Campos, ${ }^{1}$ Marcela Yataco, ${ }^{1}$ Carlos Medina, ${ }^{1}$ Kheyd Chávez, ${ }^{1}$ Richard Caballero, \\ ${ }^{1}$ Pool Flores, ${ }^{1}$ Antonio Rebaza, ${ }^{1}$ Gustavo Fernández, ${ }^{1}$ Silvia Paucar, ${ }^{1}$ Carlos Carrel, ${ }^{1}$ Katterine Gonzales, \\ ${ }^{1}$ Jhosselin Mallqui, ${ }^{1}$ Manuel Acuña, ${ }^{1}$ Zoila Gonzales, ${ }^{1}$ José Castillo, ${ }^{1}$ Analy Cauti, ${ }^{1}$ Ana Huayanca, ${ }^{1}$ Claudia \\ Paniagua, ${ }^{2}$ Manuel Cotrina, ${ }^{1}$ Gloria Gordillo.
}

${ }^{1}$ Laboratorio de Bioinformática y Farmacogenética (BIOPHARM), Departamento de Bioquímica, Instituto de Química Biológica, Microbiología y Biotecnología "Marco Antonio Garrido Malo", Facultad de Farmacia y Bioquímica - UNMSM, Jr. Puno, 1002, Lima, Lima,

${ }^{2}$ Departamento de cirugía en mamas y tejidos blandos, Instituto Nacional de Enfermedades Neoplásicas. Av. Angamos Este 2520, San Borja, Lima.

DOI: https://doi.org/10.33017/RevECIPeru2014.0010/

\section{Resumen}

A nivel mundial, según datos publicados por el Center for Disease Control (CDC), presenta a la neoplasia maligna como la segunda causa de muerte (185.1 óbitos en cien mil habitantes registrado en el 2011) apenas siendo superada por enfermedades cardiovasculares. En el Perú, esta situación no es ajena. La neoplasia maligna o cáncer es considerado, según un informe publicado en el 2013 por el Instituto Nacional de Enfermedades Neoplásicas (INEN), la segunda causa de muerte en la población peruana, siendo el cáncer de mama, el segundo tipo de cáncer más prevalente y de mayor mortalidad en la población femenina de todas las edades. El cáncer es un problema que aflige, en promedio, 45000 peruanos anualmente, de acuerdo al Departamento de Epidemiología de Cáncer del INEN, problema que no solo carga consigo el deterioro progresivo de la salud, sino que también impacta en el aspecto socioeconómico de cada individuo. Actualmente, $75 \%$ de los casos de diagnóstico de cáncer son detectados en estadio avanzado, lo cual empeora el pronóstico del paciente a largo plazo, y por consiguiente, conlleva a una menor calidad de vida, mayores costos de tratamiento, elevada probabilidad de recidiva y metástasis y un aumento exacerbado de la mortalidad. Por otro lado, las determinantes sociales que delimitan a la población peruana juegan un rol relevante en la progresión y desarrollo del cáncer: Disparidades socio-económicas manifestadas como falta de acceso a los servicios de salud en zonas pobres y remotas provocan un sub-diagnóstico del status del cáncer y opaca la verdadera magnitud con la cual ésta afecta a la población peruana, ocasionando que solo sea observado la punta del iceberg y no el fondo del problema. El Registro de Cáncer de Lima Metropolitana, iniciativa emprendida hace más de una década por el INEN, registra pacientes diagnosticados con cáncer con un mínimo de 8 meses de residencia en la capital peruana, registro que permite cuantificar variables epidemiológicas para el planeamiento de estrategias y políticas nacionales, pero, que no consigue reflejar la realidad nacional. Según la Organización Mundial de la Salud (OMS), el 30\% de las neoplasias malignas están asociados a factores de riesgo epidemiológicos, tales como factores modificables y no modificables, que tienden a incrementar la probabilidad de aparición de la enfermedad, básicamente relacionados con estilos de vida poco saludables. Teniendo en cuenta que la etiología del cáncer es multifactorial, de la cual, el factor genético y otros factores 
no modificables son inexorables, colocando en mayor riesgo a aquellos individuos de determinada edad y etnia con antecedentes familiares de predisposición genética de ciertos proto-oncogenes, también se debe considerar a los ya mencionados por la OMS, factores de riesgo relacionados con estilos de vida que actuarían como promotores o protectores en la aparición y desarrollo del cáncer. Por causa de lo mencionado, el principal objetivo del proyecto es determinar la asociación de estos factores de riesgo relacionados con estilos de vida con la progresión del cáncer de mama en pacientes atendidas en un establecimiento de salud nivel III. Dentro de los factores evaluados se tomaron en consideración: consumo de vitamina $\mathrm{C}$ y $\mathrm{E}$, actividad física, número de hijos, tiempo de lactancia, uso de anticonceptivos, consumo de alcohol, hábito de fumar y exposición a rayos $X$. El proyecto abordó un estudio prospectivo de casos $(n=264)$ y controles $(n=391)$ a través de entrevistas personales con participantes altamente capacitados, tanto para los casos como en los controles, para la recolección y análisis de los datos realizados. Finalmente, los resultados muestran que ciertos factores modificables en la población peruana como consumo de vitamina $\mathrm{C}$ y $\mathrm{E}$, actividad física y el número de hijos $(p<0.05)$ estarían actuando como factores protectores frente al desarrollo del cáncer y en contraparte, enfermedades benignas previas y antecedentes familiares $(p<0.05)$ se presentan como factores que promueven la aparición de cáncer.

Descriptores: Cáncer de mama, determinantes sociales, factores genéticos, factores de riesgo relacionados con estilos de vida, factores modificables, estudio prospectivo de casos y controles

\section{Abstract}

Worldwide, according to data published by the Center for Disease Control (CDC), malignancy neoplasms are presented as the second leading cause of death (185.1 deaths per hundred thousand inhabitants described in 2011) being barely surpassed by cardiovascular diseases. In Peru, this situation is not unfamiliar. Malignant neoplasm or cancer is considered, according to a report published in 2013 by the National Institute of Neoplasm Diseases (INEN), the second leading cause of death in the Peruvian population, with breast cancer, being the second most prevalent type of cancer and with high rates of mortality among women of all ages. Cancer is a problem that afflicts, in average, 45,000 Peruvians annually, according to the Department of Cancer Epidemiology of INEN, charging the burden of being a problem not only related to the progressive deterioration of health, but also impacting on the socio-economic aspect of each individual. Currently, $75 \%$ of cases of cancer diagnosis are detected at an advanced stage, which worsens patient prognosis in long-term, and therefore, leads to a lower quality of life, increased treatment costs, high likelihood of recurrence and metastases and an exacerbated increase in mortality. On the other hand, the social determinants that define the Peruvian population play an important role in the progression and development of cancer: Socio-economic disparities manifested as lack of access to health services in poor and remote areas result in a sub-diagnosis cancer status and blur the true extent to which it affects the Peruvian population, what causes only looking the tip of the iceberg and not the core problem. The Cancer Registry of Lima, initiative launched more than a decade ago by the INEN, registers patients diagnosed with cancer with at least 8 months of residence in the Peruvian capital, this registry quantifies epidemiological data in order to plan national strategies and policies, but which fails to reflect the national reality. According to the World Health Organization (WHO), 30\% of cancers are associated with epidemiological risk factors, such as modifiable and non-modifiable factors that tend to increase the likelihood of developing the disease; those factors are primarily related with unhealthy lifestyles. Given that cancer etiology is multifactorial, in which the genetic factor and non-modifiable factors are inexorable, placing a greater risk to individuals of a certain age and ethnicity with a family history of genetic predisposition on certain protooncogenes, it should also be considered the aforementioned WHO idea, risk factors related to lifestyles that act as promoters or protectors in the onset and development of cancer. Because of the above, the main objective of the project is to determine the association of these risk factors related to lifestyle with the progression of breast cancer in patients treated in a health facility level III. Among the evaluated factors were taken into account: consumption of vitamin $\mathrm{C}$ and $\mathrm{E}$, physical activity, parity, duration of breastfeeding, contraceptive use, alcohol consumption, smoking and exposure to X-ray. The project addressed a prospective cases $(n=264)$ and controls $(n=391)$ project through personal interviews with highly trained participants, for both cases and controls, for the collection and analysis of data carried. Finally, the results show that certain modifiable factors in the Peruvian population such as intake of vitamin $C$ and $E$, physical activity and the number of children ( $p$ 
$<0.05)$ would act as protective factors against the development of cancer and in contrast, previous benign diseases and family history $(\mathrm{p}<0.05)$ are presented as factors that promote the development of cancer.

Keywords: Breast cancer, social determinants, genetic factors, lifestyles associated-risk factors, modifiable factors, prospective study of cases and controls

\section{Introducción}

El cáncer es un flagelo social que, anualmente, afecta a 45000 peruanos en promedio, adscritos al Registro de Cáncer de Lima Metropolitana, según el Departamento de Epidemiología y Estadística de

Cáncer del Instituto Nacional de Enfermedades Neoplásicas (INEN). INEN, por medio de dicho registro, ha ratificado tasas de incidencia y mortalidad de 150.7 y 78.3 por cien mil habitantes en la Ciudad de Lima, respectivamente. De no contar con las medidas ni programas preventivos necesarios que colaboren a reducir significativamente la incidencia de Cáncer en el Perú, para el 2025 existirán 50000 casos nuevos al año, siendo el cáncer de mama la 2 da causa de muerte más frecuente en mujeres. Esta tendencia hacia el desarrollo de programas de prevención ha sido muy bien desarrollada en otros países; y todo ello surge en base al estudio de los factores de riesgo asociados a la aparición de cáncer. Evidentemente, el factor genético es inexorable, pero factores sociodemográficos, ambientales o de hábitos como el consumo de alcohol y tabaco, de salud familiar y personal como el número de alumbramientos, los años de lactancia materna, el inicio de la menopausia, el índice de masa corporal, el uso de anticonceptivos, entre muchos más poseen repercusión significativa en la etiopatogenia del cáncer de mama en mujeres [Wrensch et al., 2003], los cuales tras ser evaluados se pueden dividir en desencadenantes o preventivos del cáncer de mama. Estos factores son evaluados y contrastados en diferentes grupos etarios, grupos étnicos u otro tipo de caracterización, a través de indicadores epidemiológicos que otorguen el poder estadístico suficiente con el objetivo de correlacionar factorincidencia de cáncer de mamá.

Un estudio realizado en México en el Centro Médico Nacional Siglo XXI de Chihuahua demuestra que, de las 100 pacientes evaluadas, el $66 \%$ presentó uso de anticonceptivos orales, en promedio durante 3,4 años entre los 20 a 25 años, asimismo la exposición a este factor en este grupo etario durante 5 años o más aumenta drásticamente la incidencia de cáncer de mama antes de los 45 años. Junto a este último factor podemos enmarcar a la obesidad debido a que el abundante tejido adiposo y la ingestión de grasas o derivados lipídicos en mujeres obesas conlleva de forma metabólica, a un depósito de androstenediona, un derivado esteroideo que al hidroxilarse en C3 y demetilarse en C6 produce estrona (una de las 3 más importantes hormonas estrogénicas), fácilmente oxidable a Estradiol en C17. La sobreproducción de hormonas estrogénicas, adicionalmente el empleo de la terapia de reemplazo hormonal confiere una elevada probabilidad de carcinogénesis [Christopher I. Li et al. 2003]. El embarazo ejerce un efecto protector sobre el epitelio mamario, al influir directamente en la diferenciación celular y en la reducción de hormonas estrogénicas, en el estudio Mexicano previamente mencionado, el $23 \%$ de los pacientes tuvieron su primer embarazo después de los 30 años [Salas, Ivonne et al. 2006].

Las diferentes cifras de incidencia en los países son establecidas por la frecuencia de aparición de los factores de riesgo. En el periodo 1998-2002, la tasa de incidencia fue de 81 y 41 por cada 100000 mil habitantes en Canadá y Hong Kong respectivamente [Curado, M. P. et al. 2007], mientras que en el Perú oscilaba cerca a los 100 por cada 100000 mil habitantes según una tendencia sugerida por el Dpto. de Epidemiología y Estadística de Cáncer - INEN. Los estilos de vida como factor de riesgo marcan gran diferencia al compararlos entre dos etnias que difieran en hábitos alimentarios, educación sexual u otros. Existe un $37 \%$ de riesgo de mortalidad menor en la población China que en población canadiense; en cambio cuando inmigrantes Asiáticos viven por un periodo mayor a 10 años en Países industrializados como Estados Unidos o Canadá, la tasa de incidencia entre ambas poblaciones, china y norteamericana, demuestra ser prácticamente igual, lo cual indica que la "occidentalización" por parte de la población China es un grupo de factores de riesgo, que consiste en la adaptación del Poblador Chino a la cultura occidental en alimentación y hábitos principalmente.

Existe una diferencia $(p<0.001)$ del $4.5 \%$ en incidencia de cáncer entre los pobladores Caucásicos-Canadienses (76.6\%) y los Chinos recién migrados hacia Canadá $(72.1 \%)$ respecto al hecho de lactancia materna hacia el hijo. Esta diferencia se torna abrupta en el uso de anticonceptivos orales (ACO), 45.1\% ( $p<0.001$ ). Solo el $40.7 \%$ de las 421 
chinas recién migradas emplearon ACO durante 2.1 años en promedio, mientras que el $85.8 \%$ de las 413 Caucásicas emplearon ACO durante 3.6 años ( $p<$ 0.001) [Carolyn y Tam et al. 2010]. Cabe resaltar que conocer el trasfondo molecular ayuda al Profesional de la Salud a la elaboración de Terapias preventivas "dirigidas" con una alta probabilidad de ser eficaz, y que aún existe la necesidad, afirma el INEN, de proyectos de investigación que permitan descubrir cómo se relacionan estos factores de riesgo con la incidencia de cáncer.

El presente proyecto tiene por objetivo determinar los factores de riesgo relacionados con estilos de vida asociados a la aparición del cáncer de mama en una población atendida en un establecimiento de salud nivel III en Lima Metropolitana.

\section{Materiales y métodos}

Se realizó un estudio prospectivo observacional y de corte analítico, de casos y controles sobre los principales factores de riesgo relacionados con estilos de vida asociados con la aparición y progresión de cáncer de mama. Fue diseñado y validado un cuestionario dirigido tanto para los casos y controles, el cual fue construido en base a literatura que da sustento y validez científica a cada factor en relación a la patología en estudio.

Fueron establecidos criterios de inclusión/exclusión para la selección tanto de los casos como los controles, así como la aprobación del participante al Término de Consentimiento Libre y Esclarecido para formar parte del estudio.

La colecta de datos de los controles $(n=391)$ se realizó de forma aleatoria a mujeres peruanas que cumplieran con los criterios de inclusión. Las entrevistas fueron llevadas a cabo en diversos lugares de Lima Metropolitana en donde el entrevistado se sintiera menos presionado de la mejor forma posible, y evitándose algún sesgo de ubicación geográfica.

La reunión de datos de los casos $(n=264)$ se realizó en las inmediaciones del Instituto Nacional de Enfermedades Neoplásicas (INEN) del Perú. Fue solicitada previamente un convenio académico con el Departamento de Investigación del INEN que permitiese el ingreso de estudiantes al instituto. Tras aprobación del Comité de Investigación, fue permitido el acceso del equipo de investigación para la realización de las encuestas a pacientes ambulatorios y hospitalizados que cumpliesen los criterios de inclusión.

Finalmente, los factores de riesgo en estudio fueron categorizados para facilitar el análisis. Los resultados de las encuestas fueron analizadas a través del cálculo de Odds Ratio $(\mathrm{OR})$, teniendo en consideración aquellos resultados estadísticamente significativos $(p<0.05)$.

\section{Resultados y discusión}

Tras finalizar la recolecta de datos, se realizó el análisis estadístico que brindó como resultado el grado de asociación o causalidad de los factores de riesgos relacionados a estilos de vida asociados con la aparición y progresión de cáncer de mama en la población femenina de Lima Metropolitana - Perú de la siguiente manera:

Factores de riesgo no modificables no relacionados con estilos de vida asociados de forma negativa y actuando como promotores de la aparición y progresión de cáncer de mama en población femenina de Lima Metropolitana:

- No modificable. La presencia de enfermedades benignas previas en tejido mamario incrementa la probabilidad de desarrollar cáncer de mama: $\mathrm{OR}=3.01 ;(1.72$ - 5.28)

Factores de riesgo, tanto relacionados con estilos de vida así como no modificables, asociados de forma positiva y actuando como agentes protectores frente a la aparición y progresión de cáncer de mama en población femenina de Lima Metropolitana:

- No modificable. La edad de inicio de menopausia antes de los 55 años presenta un factor protector: OR $0.4(0.24-0.66)$

- Modificable. El consumo de vitamina E y sus fuentes alimenticias por al menos 3 veces por semana presenta un efecto protector. OR = $0.42 ;(0.27-0.65)$.

- Modificable. El consumo de vitamina $\mathrm{C}$ y sus fuentes alimenticias por al menos 3 veces por semana presenta un efecto protector. $\mathrm{OR}=$ $0.38 ;(0.23-0.61)$. 
- Modificable. La actividad física moderada actúa como efecto protector contra el cáncer de mama. $\mathrm{OR}=0.42 ;(0.27-0.65)$.

- Modificable: El hecho de haber salido embarazada y dar a luz niños vivos también es un factor protector. Las madres que han dado a luz a un solo niño tienen los siguientes resultados. OR: $0.36,(0.18-0.70)$. Las madres que presentan más de un niño nacido arrojan los siguientes resultados como factor protector. $\mathrm{OR}=4.38 ;(2.03-9.45)$

\section{Conclusiones}

El estudio demuestra que 4 de los factores estudiados se presentan como factores de riesgo modificables con probabilidad de manifestarse como un agente protector frente a la aparición y progresión del cáncer de mama. Tanto el consumo de vitamina $\mathrm{C}$ y $\mathrm{E}$, la actividad física y el número de hijos influencian de forma positiva y disminuyen las probabilidades de desarrollar cáncer en pacientes peruanas.

Nuestros esfuerzos son retroalimentados por resultados que autores anteriores han relatado en grandes poblaciones extranjeras, y por lo tanto, procuramos alinearnos a los esfuerzos vigentes para reforzar las acciones de salud global para fomentar un abordaje preventivo del cáncer, y aumentar la posibilidad de un diagnóstico precoz en estas poblaciones más susceptibles.

La perspectiva a largo plazo es tornar al cáncer en una patología con abordaje preventivo a través del desarrollo de algoritmos clínicos que nos permitan encaminar mejor al paciente disminuir, disminuir la morbi-mortalidad, así como predecir el curso de la enfermedad. Sin embargo, esto no resulta de la labor de un grupo de investigación, sino, del accionar conjunto de institutos, universidades, establecimientos de salud y, por supuesto, de la población. Por ello, es importante el fomento de la identificación de factores de riesgo que permitan sensibilizar al público objetivo, de manera que se combatan aquellas altas tasas epidemiológicas que el cáncer viene alcanzando en las últimas décadas.

\section{Agradecimientos}

El estudio realizado por el laboratorio de bioinformática y farmacogenética, conformado por el grupo de estudio "BIOPHARM" de la Facultad de Farmacia y Bíoquímica, agradece infinitamente el apoyo de su fundadora, maestra y orientadora, que dio tutela y motivación, además de guiarnos en el planeamiento y desarrollo del presente estudio, Dr.

Gloria Gordillo Rocha

Agradecemos al Vicerrectorado de Investigación de la Universidad Nacional Mayor de San Marcos por el apoyo financiero brindado para la ejecución del proyecto.

De la misma manera, el Departamento de Investigación del Instituto Nacional de Enfermedades Neoplásicas recibe nuestro mayor agradecimiento por permitirnos realizar este estudio en colaboración con ellos.

Asimismo, un agradecimiento personal para nuestro colaborador clave, Dr. José Manuel Cotrina Concha, Director Ejecutivo del Departamento de Cirugía en Mamas y Tejidos Blandos del INEN, el cual fue fundamental para la conclusión del presente proyecto, $y$ con el cual planeamos continuar con las investigaciones.

Finalmente, agradecemos la colaboración de todos los integrantes del grupo "BIOPHARM" por el alto grado de profesionalismo que han mostrado en el transcurso del proyecto, por la perseverancia que mantuvieron en el mismo a pesar de que abordar personas siempre es complicado y por el amor a la ciencia que todos hemos cultivado.

\section{Referencias}

[1] M. Wrensch, et al, Risk factors for breast cancer in a population with high incidence rates, Breast Cancer Research, 5 (2002), No 4, R88 - R102.

[2] C. I., Li; J. R., Daling; K. E., Malone. J. Clin. Onco, 21 (2003), No 1, 28 - 34.

[3] I, Salas; B. L.; Ramirez; Z.; E., Apodaca. CIMEL, 11 (2006), No 2, 62 - 66

[4] M. P., Curado; B., Edwards; H. R. Shin; H., Storm; J., Ferlay; M., Heanue; P. Boyle. Cancer Incidence in Five Continents. IARC Scientific Publication, International Agency for Research on Cancer, WHO, IX (2007), No 160. 
[5] C. Y., Tam; L. J., Martin; G., Hislop; A. J., Hanley; S., Minkin; N. F., Boyd. Risk factors for breast cancer in postmenopausal Caucasian and Chinese-Canadian women. Breast Cancer Research, 12 (2010), R2, 1 - 12.
E-mail: antonybcampos@usp.br, myatacolazaro@gmail.com, carlosmedsa@gmail.com, hattie.26.93@gmail.com, gordillogloria@gmail.com 